\title{
Common nutritional deficiency with unusual site of presentation
}

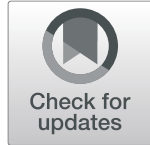

Kandan Balamurugesan ${ }^{1}$, Rajangam Ponprabha ${ }^{2}$ and Prem Davis ${ }^{3,4^{*}}$ (ID

\begin{abstract}
Background: Pellagra is a nutritional disorder due to deficiency of vitamin B3 (niacin) which is characterized by dermatitis, dementia, diarrhea, and death. The most common site of dermatitis in pellagra is the Casal necklace; however, in this case, the presentation of dermatitis is in unusual sites. Therefore, mucocutaneous signs play a major role in diagnosing the condition. The clinical features of pellagra begin with gastrointestinal symptoms followed by neurological symptoms which are subtle and non-specific. But if misdiagnosed and untreated, it can progress to death from multiorgan failure.

Case presentation: A 46-year-old Indian farmer, chronic alcoholic of 20 years duration, presented to the hospital with the complaints of darkening and thickening of the skin over the left upper chest and shoulder, epigastric region, left arm and forearm, and right arm and forearm for a period of 4 months with mild cognitive decline. His blood parameters revealed moderate anemia with microcytic hypochromic blood picture and decreased MCV, $\mathrm{MCH}$, and $\mathrm{MCHC}$ values. A diagnosis of pellagra was built clinically after exclusion of other causes and has been treated with nicotinamide and had drastic improvement on follow-up.

Conclusion: The unusual site of pellagra presentation is rare; however, the uncommon presentation of a common disease is always to be considered for diagnosing the disease at the early stage. The neuropsychiatric symptoms of the pellagra in a known case of chronic alcoholic are not well defined, and if untreated, it may progress to stupor and coma and ultimately death. Hence, appropriate diagnosis and earlier management helps in the prevention of various life-threatening complications of any disease.
\end{abstract}

Keywords: Pellagra, Niacin, Alcoholic, Dermatitis, Anemia

\section{Background}

Until twentieth century, pellagra was a major cause of death associated with malnutrition and extreme poverty. Now it has been eradicated in most of the countries due to remarkable enhancement in public health and nutrition. But still, cases are occurring among alcoholics, drug abusers, food faddists, and among homeless people [1, 2]. Don Gaspar Casal first described pellagra in 1735 as "mal de la rosa," and was later renamed as pellagra in Italy from "pelle agra" which means rough skin which

\footnotetext{
* Correspondence: premdavis93@gmail.com

${ }^{3}$ Health Centre, Central University of Tamil Nadu, Thiruvarur, Tamil Nadu, India

${ }^{4}$ Department of ENT, Sri Venkateswaraa Medical College Hospital and Research Centre, Ariyur, Puducherry, India

Full list of author information is available at the end of the article
}

was noted among the Spanish poor people [3]. Pellagra is a nutritional disorder that occurs due to the deficiency of niacin (vitamin B3), which mainly affects the skin, the gastrointestinal tract, and the nervous system. It is classically characterized with 4Ds, namely dermatitis, dementia, diarrhea, and death [4]. The incidence of pellagra in India is not known due to underreporting of cases. The diagnosis is usually made on clinical characteristics and its immediate response to treatment [5]. Pellagra is usually observed in areas of extreme poverty and is rare in many developed countries due to the availability of adequate nutritional foods; majority of cases occur in chronic alcoholics. The pellagra is an easily treatable condition which can be fatal if not recognized, it should be considered in patients with classical clinical 
signs and symptoms, and treated with appropriate dose of vitamin B3, it will result in complete resolution of skin manifestations as well as symptoms. We report a case of pellagra in a farmer in the background of chronic alcohol consumption with cognitive decline, was diagnosed and treated with vitamin B3, and had complete recovery.

\section{Case presentation}

A 46-year-old male, farmer by occupation, presented to the hospital with complaints of darkening and thickening of the skin over the left upper chest and shoulder, epigastric region, left arm and forearm, and right arm and forearm for a period of 4 months. The patient initially developed darkening of the skin over the left shoulder which gradually involved other sun-exposed regions of the body as he is a farmer. He also complained of ulcer over the angle of the mouth for the period of 1 month. The lesions were associated with itching and burning sensation on exposure to sunlight. $\mathrm{He}$ is a chronic alcohol consumer for the past 20 years and consumes $250 \mathrm{ml}$ of alcohol daily. He denied any changes in the behavior, and there is no history of memory loss, abdominal distension, pedal edema, and diarrhea. Bowel and bladder habits were normal. There is no history suggestive of other nutritional deficiencies like perioral numbness, foot pain, ataxia, easy bruisability, and join pain.

On examination, the patient is conscious, alert, and oriented. On general physical examination, the patient has pallor with angular cheilitis (Fig. 1). The patient did not have any signs of glossitis, angular stomatitis, cheilosis, bitot spots, corneal xerosis, xeropthalmia, and bleeding gums. The Mini-Mental State Examination was found to be 24/30 indicating a mild cognitive decline. On examination, multiple well-defined macules predominantly involve the photo-distributed areas like the left

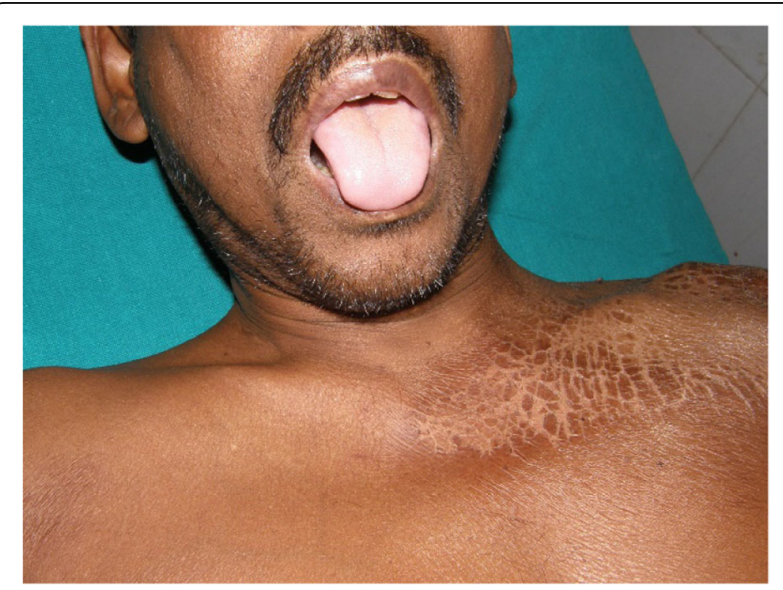

Fig. 1 Pale tongue with angular cheilitis shoulder, left upper back, epigastric region, and also over the other sun-exposed areas like the right arm and forearm and left arm and forearm (pellagra gauntlet) with a dry overlying surface with a sharp demarcation between involved and healthy skin (Fig. 2). The systemic examination is found to be normal.

His investigation revealed moderate anemia with microcytic hypochromic blood picture and decreased $\mathrm{MCV}, \mathrm{MCH}$, and $\mathrm{MCHC}$ values. Other parameters in the complete blood count were within normal limits. On iron studies, he was found to have low serum iron and low serum iron storage capacity with increased TIBC as in Table 1. Although he is a chronic alcoholic for 20 years, his liver function and renal function tests were within normal limits. HIV and ELISA were non-reactive.

Upper GI endoscopy was done to rule out other causes of anemia and malabsorption related malnutrition like esophageal web/varices and carcinoma esophagus. However, it is not contributory neither for esophageal web/ varices nor esophageal carcinoma. The serum niacin estimation could not be done due to its non-availability.

Based on the history and classical clinical findings of photosensitive, anemia, angular cheilitis, glossitis, and mild cognitive impairment, the diagnosis of pellagra was made. However, the definitive laboratory investigation for the diagnosis of pellagra, serum niacin level, was not carried out in our case. Based on the history and clinical findings of photodermatitis with mild cognitive decline and with background of chronic alcoholism, the diagnosis of pellagra was made. The patient was started on 300 $\mathrm{mg}$ of nicotinamide thrice a day with iron and other multivitamin supplements. The patient improved rapidly after the commencement of medication with resolution of skin lesions in a period of 4 weeks. The improvement of the symptoms with nicotinamide supports our clinical diagnosis (Fig. 3). The patient was advised to consume food rich in niacin like nuts especially groundnut, legumes, fish, egg, meat, and vegetables. And the patient is on regular follow-up.

\section{Discussion}

The pellagra is a multisystem chronic disease affecting the skin, the gastrointestinal system, and the nervous system. It occurs due to the deficiency of nicotinic acid (niacin or vitamin B3) or its precursor tryptophan [6]. The deficient vitamin plays an important role in the metabolism of carbohydrate, fats, proteins, alcohol, detoxifies various drugs, reactive oxygen species, and in cell signaling and DNA repair pathway [7]. A thorough review of niacin metabolism is necessary to understand the pathophysiology of pellagra. Niacin can be obtained directly from the food or synthesized from tryptophan; this synthesis requires vitamins B2 and B6 as cofactors. Dietary niacin is mainly in the form of nicotinamide 


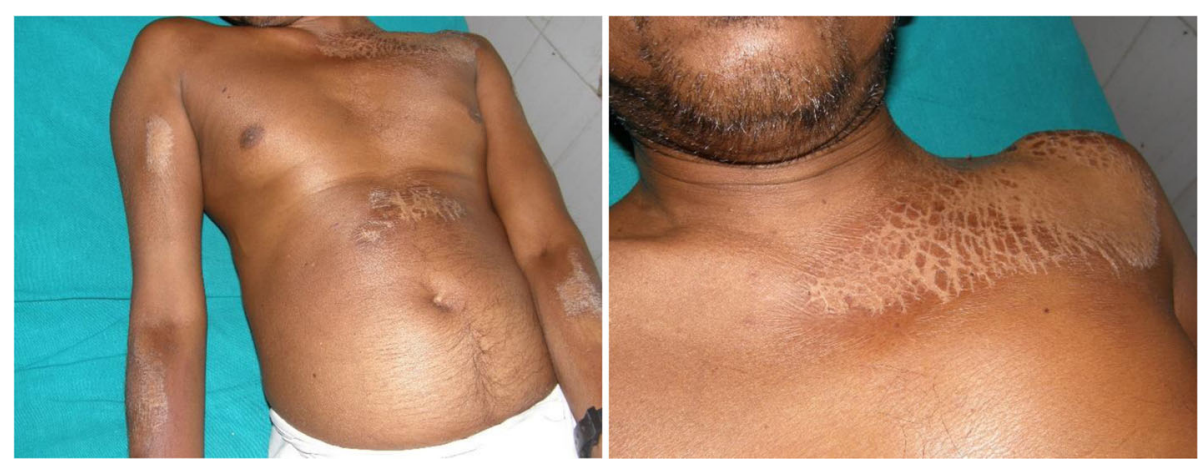

Fig. 2 Multiple well define macules predominantly involving the photo distributed areas like arm, forearm, left shoulder and epigastric region with dry overlying surface

adenine nucleotide (NAD) and NADPH. These molecules undergo hydrolysis in the intestinal lumen to form nicotinamide, which can be converted to nicotinic acid by intestinal bacteria or absorbed directly into the bloodstream. Nicotinamide and nicotinic acid are then reincorporated as a component of coenzymes NAD and NADP, which in turn intervene in essential oxidationreduction reactions. The ubiquitous presence of these coenzymes explains the multiorgan afflictions associated with pellagra. Tissues with high energy requirements such as the brain or high turnover rates such as gut or skin are primarily affected $[5,8]$. The exquisite photosensitivity seen in pellagra may result from a deficiency of urocanic acid and/or cutaneous accumulation of kynurenic acid that may induce a phototoxic reaction [5, 9]. The recommended daily allowance of niacin is 15$20 \mathrm{mg} /$ day [10]. The pellagra is divided into primary pellagra and secondary pellagra. The primary subtype mainly occurs due to dietary deficiency of niacin or tryptophan predominantly seen in people whose staple diet consists of maize. The secondary pellagra is caused by the conditions that are interfering with the metabolism or absorption of niacin or tryptophan which includes chronic alcoholism, anorexia nervosa, ileo colitis, prolonged diarrhea, Hartnup's disease, HIV, and carcinoid syndrome. Drugs like azathioprine, 5-flurouracil, isoniazid, phenobarbitone, and 6-mercaptopurine also lead to pellagra $[6,9]$. The clinical features of pellagra begin with gastrointestinal symptoms followed by neurological symptoms which are subtle and non-specific. But if untreated, it can progress to death from multiorgan failure [7]. Therefore, mucocutaneous signs play a major role in diagnosing the condition.

An acute, symmetrical, well-demarcated, tender erythematous eruptions occur at the site of sun exposure mimicking sunburn. Later, these lesions turn into hyperkeratotic, dry scaly, parchment-like, and pigmented lesions with yellow brown hue [5]. The healing of the lesions starts centrifugally with desquamation at the center and persistence of erythema at the margins. The

Table 1 Blood investigations

\begin{tabular}{lll}
\hline Test & Test values & Normal range \\
\hline Complete clood count & 7 & $13.5-17.5$ \\
$\mathrm{Hb}(\mathrm{g} / \mathrm{dL})$ & 5710 & $4000-11,000$ \\
Total leukocyte count (cells/cumm) & $65,32,1,2$ & $50-65,30-60,0-2,0-5$ \\
Differential leukocyte count (P, L, M, and E) & 60 & $76-96$ \\
MCV (ft) & 21 & $27-31$ \\
MCH (PG) & 25 & $32-36$ \\
MCHC (\%) & 2.4 & $3.5-5.5$ \\
Total RBC count million cells/cumm & 2.60 & $1.5-4.0$ \\
Total platelet count lakhs cells/cumm & & $60-170$ \\
Iron study & 45 & $240-450$ \\
Serum iron (mcg/dL) & 540 & $20-50$ \\
Total iron binding capacity (mcg/dL) & 12 & \\
Transferrin saturation (\%) & \\
\hline
\end{tabular}



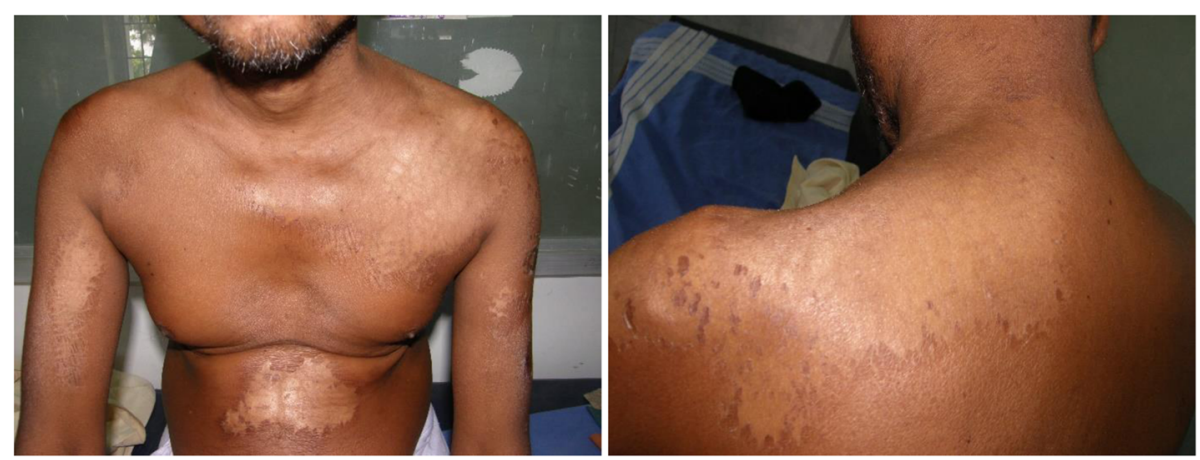

Fig. 3 Improvement of the skin lesions after the commencement of the treatment

typical lesion occurs around the nape of the neck termed as Casal's necklace and the erythema occurs recurrently over the sun-exposed area. The gastrointestinal symptoms include diarrhea, nausea, vomiting, decreased appetite, gastritis, and achlorhydria [5]. The neuropsychiatric symptoms of pellagra are not well defined and frequently confounded with other central nervous system disorder. The symptoms like headache, irritability, poor concentration, apathy, depressed mood, psychomotor unrest, and ataxia will occur, and these symptoms may progress to stupor and coma [11]. Serum niacin levels are used to estimate the levels of $\mathrm{N}$-methyl nicotinamide, which is used in confirming the disease [12]. Many times, the diagnosis is made on the basis of clinical examination and confirmed by rapid response to oral nicotinamide [7]. The histopathology of the skin usually will show nonspecific features like hyperkeratosis, parakeratosis, pallor of the upper epidermis, capillary dilatation, dermal edema, and perivascular lymph histiocytic infiltrate [7].

Since, the pellagra is due to the deficiency of the niacin. The main stay of the treatment is to replenish the store of niacin in the body. Niacinamide is preferred over nicotinic acid in the treatment of pellagra. Because the nicotinic acid in higher doses may precipitate flushing, burning, and itching. The World Health Organization recommends nicotinamide as $300 \mathrm{mg}$ orally or $100 \mathrm{mg}$ parentally in divided doses with the maintenance dose of $50-100 \mathrm{mg} /$ day after the disappearance of the neurological symptoms [13]. Most of the patients with the diagnosis of pellagra are also deficient with other nutrients, so a high protein diet with other B-complex vitamins are recommended for the patients [14]. Dietary advice with niacin-rich foods like meat, poultry, fish, dry beans, mushrooms, nuts, dairy products, and eggs, legumes, fish, vegetables, and lifestyle modifications like abstinence from alcohol, avoidance of maize, and photoprotection are encouraged [15].

\section{Conclusion}

Pellagra is a multisystem disease of the skin, the gastrointestinal system, and the nervous system. It occurs due to the deficiency of nicotinic acid or its precursor tryptophan. The classical skin presentation will not be in all of the cases. So, the clinicians should be oriented with the knowledge of the uncommon presentation of the pellagra as described in this patient for the early diagnosis and treatment.

\section{Acknowledgements \\ Not applicable}

\section{Authors' contributions}

Author 1 "KB" carried out the conception and literature review, analyzed and interpreted the patient's data, and diagnosed the patient. Author 2 "RP" contributed in the laboratory work and critical revision of the draft and shared in the final manuscript writing. Author 3 "PD" carried out conception, writing, and editing the manuscript for submission. All the authors read and approved the final manuscript.

\section{Funding}

Nil.

\section{Availability of data and materials \\ Available}

Ethics approval and consent to participate Not applicable

\section{Consent for publication}

A written informed consent to publish information was obtained from the study participant.

\section{Competing interests \\ None}

\section{Author details}

${ }^{1}$ Department of Medicine, Jawaharlal Institute of Postgraduate Medical Education and Research, Puducherry, India. ${ }^{2}$ Department of Pediatrics, Government Villupuram Medical College, Villupuram, Tamil Nadu, India. ${ }^{3}$ Health Centre, Central University of Tamil Nadu, Thiruvarur, Tamil Nadu, India. ${ }^{4}$ Department of ENT, Sri Venkateswaraa Medical College Hospital and Research Centre, Ariyur, Puducherry, India.

Received: 21 November 2020 Accepted: 16 December 2020

Published online: 15 January 2021

\section{References}

1. Usman AB, Emmanuel P, Manchan DB, Chinyere A et al (2019) Pellagra, a reemerging disease: a case report of a girl from a community ravaged by insurgency. Pan Afr Medial J 33:195

2. Galimberti F, Mesinkovska N (2016) Skin finding associated with nutritional deficiencies. Cleve Clin J Med 83:731-739 
3. Arif T, Adil M, Amin SS (2018) Pellagra: an uncommon disease in the modern era- a case report. J Pakistan Assoc Dermatol 28(3):360-363

4. Gupta SK, Arora AK, Sood N, Kaur S (2014) Pellaagra revisited. Indian Dermatol Online J 5:525

5. Karthikeyan K, Thappa DM (2002) Pellagra and skin. Int J Dermatol 41:476-481

6. Wan P, Moat S, Anstey A (2011) Pellagra: a review with emphasis on photosensitivity. Br J Dermatol 164:1188-1200

7. Thornton AM, Drummond CJ (2014) An unexpected case of pellagra. Med J Austr 200(19):93

8. Jen M, Shah KN, Yan AC (2008) Cutaneous changes in nutritional disease. In: Wolff K, Goldsmith LA, Katz SI, Gilchrest BA, Paller AS, Leffel DJ (eds)

Fitzpatrick's dermatology in general medicine, 7th edn. New York: McGraw Companies. pp 1209-1211

9. Hegyi J, Schwartz RA, Hegyi V (2004) Pellagra: dermatitis, dementia and diarrhea. Int J Dermatol 43:1-5

10. Segula D, Banda P, Mulambia C, Kumwenda JJ (2012) Case report - a forgotten dermatological disease. Malawi Med J 24:19-20

11. Brown TM (2010) Pellagra: an old enemy of timeless importance. Psychosomatics. 51:93-97

12. Dhawan AK, Grover C, Sharma S (2014) Phenytoin induced pellagroid dermatitis. Indian J Dermatol 76:103-115

13. Garg G, Khopkar U (2011) Ethionamide induced pellagroid dermatitis resembling lichen simplex chronicus. A report of two cases. Indian J Dermatol Venereol Leprol 77:534

14. Kaimal S, Thappa DM (2010) Diet in dermatology: revisited. Indian J Dermatol Venereol Leprol 76:103-115

15. De Oliveira AP, Antonio S (2020) Pellagra: a report of two cases. Med Case Rep 6(2):138

\section{Publisher's Note}

Springer Nature remains neutral with regard to jurisdictional claims in published maps and institutional affiliations.

\section{Submit your manuscript to a SpringerOpen ${ }^{\circ}$ journal and benefit from:}

- Convenient online submission

- Rigorous peer review

- Open access: articles freely available online

- High visibility within the field

Retaining the copyright to your article

Submit your next manuscript at $\boldsymbol{\nabla}$ springeropen.com 